\title{
Research on Pre-Job Training in the Top-Three Hospital in Chongqing -Based on Social Role Theory
}

\author{
Tu Qing1, Huang Li² \\ ${ }^{1}$ The School of Public Health and Management, Center for Medicine and Social Development, The Innovation \\ Center for Social Risk Governance in Health, Chongqing Medical University, Chongqing, China \\ ${ }^{2}$ The First Affiliated Hospital of Chongqing Medical University, Chongqing, China \\ Email:691836990@qq.com
}

Received 6 January 2016; accepted 24 February 2016; published 29 February 2016

Copyright $@ 2016$ by authors and Scientific Research Publishing Inc.

This work is licensed under the Creative Commons Attribution International License (CC BY). http://creativecommons.org/licenses/by/4.0/

(c) () Open Access

\section{Abstract}

The aim of the paper is to describe the basic information of new staffs in the top-three hospital in Chongqing, analyze their needs and evaluation for pre-job training, and establish curriculums and training methods which are combined with social role theory. Questionnaire is used to survey new staffs who participated in pre-job training in the first half year in 2014. This questionnaire is anonymous and collected immediately. Excel is used to enter and analyze data. First, the majority of new staffs are doctors and masters who belong to clinical department. What is more, most are females. Second, most of staffs are satisfied with this training in overall management, time management, and training content. However, a small number of staffs think training methods are monotonous. Curriculums contents should be designed according to the characteristic of jobs, education, and major. Training methods should be interactive. Moreover, hospitals need to intensify information feedback to improve pre-job training from multi-aspects.

\section{Keywords}

Top-Three Hospital, Social Role, Pre-Job Training

\section{Introduction}

Nowadays, new staff pre-job training is an important task for hospital's human resources management and gets great attention in domestic medical institutions [1] [2]. The significance of pre-job training is to strengthen the culture, promote the construction of hospital culture, and improve the quality of staff and the sense of mission. 
The new workers from school or other work units may face many problems, such as adapting to the new environment, changing their role identity and adaptation of rules and regulations. The success of pre-job training can help them quickly understand the history and culture of hospital, the rules and regulations, the hospital's overall development plan and the future development direction of the hospital, and the business philosophy and core values of the hospital [3]. These not only have contribution to new employees at work for personal self-positioning and career development planning, but also help them more quickly adapt to the hospital environment. Based on pre-job training for new staff satisfaction analysis and combined with the social role theory, this study was designed to research on new staff who admitted in the first half 2014 in a large hospital to explore and improve the quality of pre-service training of hospital and provide reliable scientific evidence to make recommendations and thinking [4].

\section{Social Role Theory}

Social role is defined by people's social status, showing the total pattern consistent with socially desirable behavior and attitudes. Social roles include three meanings: first, it is a social behavior. Social behavior is reflected in every specific social roles; second, it is defined by people's social status and identity of the decision, a true reflection of the role of behavior individuals in the group life and social relations system location [5] [6]; third, it is in line with social expectations, according to the social norms of the provisions of the act, the responsibilities and obligations to act. Any kind of social behavior, not only reflects the social status and identity of role players, and reflects the relationship between the individual psychological, behavioral and group psychology, behavior and social norms between. Individual identity in particular social relations which reflect the individual position in social relations, it is a collection of social functions, rights and obligations of individuals. Each social identity are accompanied by specific codes of conduct and behavior patterns, when an individual produces for its own identity under the social norms and behavior patterns, they act as a role.

From different point of view, social roles can be divided into many types:

Firstly, it is defined as ascribed roles and the role of self-induced. Ascribed role is home role, which is built on the basis of blood factors, genetic and other congenital or physiology on social roles. For example, a person from birth has been given race, ethnicity, family background, gender roles. The so-called self-induced role, also known as self-eligible roles or achievements roles, which refers to the social role mainly through the activities and efforts of individuals obtained. The role of self-induced achieved as a result of the activities of individuals, but also the result of personal choice.

Second, it is defined as the role of conscious and unconscious role. This is based on the psychological state of people assume when doing social role distinction. The so-called role of conscious, that people assume a role at the time, clearly aware of his being charged with certain rights, obligations, aware of the people around the role of his own audience, thus trying to infect his own actions the audience around. Everyone is conscious of the role in the social life are often experienced. The so-called unconscious roles that people assume a role at the time, and did not realize that they are acting this role, but only do it by habitual behavior. In general, the current referred to, when the formation of conscious role of those reasons do not exist, it is easy to form unconsciously role [7].

In society, the role is not isolated, but linked with other roles. Such a set of interrelated, interdependent and mutually reinforcing roles is called role set. Any one person cannot just assume a certain kind of social roles, but always bear a variety of social roles, he assumed a variety of roles and always with more social roles linked to all these constitute the set of roles. Specifically, it includes the following four aspects of meaning: the role of the external manifestations of social status; the role is a set of people's rights, norms and behavior patterns obligations; the role of people in a particular position for the behavior of people's expectations; role is the basis for social groups or social organizations.

\section{Material and Method}

\subsection{Hospital Introduction}

The research is at the first "three A-level hospital”. The hospital has 35 clinical departments, 8 medical departments, 3200 beds existing establishment. Outpatient visits are 289.75 billion people in 2014. The number of discharged patients is 12.09 billion, operation in the year is 87,900 times. It is one of the key large-scale comprehensive teaching hospital which focuses on medicine, teaching, research, prevention, care and foreign health care. 


\subsection{Participants}

This study includes 92 new employees who are admitted in the first "three A-level hospital” in Chongqing. They participated the pre-job training in the first half of 2014.

\subsection{Research Content}

First, questionnaire has the basic situation for new employees, including jobs, education, and gender and so on. Second, the new overall effect on pre-job training of employees, rationality, training methods in the form of timing, arrange course content and training new employees will meet the satisfaction evaluation visits. Third, the new employees choose the pre-job training courses always a favorite. Fourth, open-ended questions, the staff of the lack of pre-job training course content and training activities suggestions and opinions.

\subsection{Methods}

Questionnaire: Totally 92.92 questionnaires are recovered, the recovery rate is $100 \%$; the number of valid questionnaires is 82 , the efficiency is $89 \%$.

Statistics: The data entry, processing and analysis of questionnaires are done by EXCEL 2003 software.

\section{Result}

Firstly, new basic situation of workers (Table 1), which can be seen from the above data were in the first half of 2014 to participate in pre-job training for new staff, there are 77 people (93.9 percent) in physicians in clinical departments, and medical technicians are 6 people (6.1\%). There was no management or care sector to participate in the pre-service training. Master are 42 (51.3\%), and 34 doctoral degree (41.4\%), six undergraduate degree (7.3\%). In addition, in those surveyed, men are 34 people (41.4\%), 48 are females (58.6\%). Their average age is 28.

Secondly, satisfaction survey (Table 2), the statistics show that the training of new workers in the six aspects of pre-job training of satisfaction evaluation. Most respondents in the pre-service training of the overall evaluation of the reasonableness of the content and timing of training courses considered to be more satisfied with these three issues. In the form of the training seminar on evaluation of problems 43.9 percent of people chose the "general" And in the new staff will meet and organize training evaluation visits were $75.6 \%$ and $79.2 \%$ of people said it was satisfied, choose "good".

Thirdly, Survey of open-ended questions:

1) "Your favorite course (limited option 5)?” This is a reflection of the new workers in different jobs and education curriculum is different preference. Most respondents were jointly selected themes of hospital cultureGlory and responsibility and Personnel topics. In these, the majority of respondents' clinical departments choose the topic, and medical departments of respondents chose more medical topics. Most new workers doctoral degrees in this issue are also at the same time select a research topic.

2) “Pre-job training courses have what it takes to increase?” On this issue respondents believe lecture content can increase review materials, curriculum content too theoretical, too single form, the curriculum can increase more case studies and scenarios simulation and role-playing.

Table 1. Basic information of 82 new staffs.

\begin{tabular}{|c|c|c|c|c|c|c|c|c|}
\hline \multirow[b]{2}{*}{ Position } & \multicolumn{2}{|c|}{ Doctor } & \multicolumn{2}{|c|}{ Postgraduate } & \multicolumn{2}{|c|}{ Undergraduate } & \multicolumn{2}{|c|}{ College } \\
\hline & Number & Percentage & Number & Percentage & Number & Percentage & Number & Percentage \\
\hline Physician & 32 & $39 \%$ & 39 & $47.6 \%$ & 6 & $7.3 \%$ & 0 & 0 \\
\hline Medical Technician & 2 & $2.4 \%$ & 3 & $3.7 \%$ & 0 & 0 & 0 & 0 \\
\hline Nurse & 0 & 0 & 0 & 0 & 0 & 0 & 0 & 0 \\
\hline Manager & 0 & 0 & 0 & 0 & 0 & 0 & 0 & 0 \\
\hline Others & 0 & 0 & 0 & 0 & 0 & 0 & 0 & 0 \\
\hline Total & 34 & $41.4 \%$ & 42 & $51.3 \%$ & 6 & $7.3 \%$ & 0 & 0 \\
\hline
\end{tabular}


Table 2. Questionnaire information.

\begin{tabular}{ccccccccc}
\hline & \multicolumn{2}{c}{ Very good } & \multicolumn{2}{c}{ Good } & \multicolumn{2}{c}{ General } & \multicolumn{2}{c}{ Bad } \\
\cline { 2 - 8 } & Number & Percentage & Number & Percentage & Number & Percentage & Number & Percentage \\
\hline Pre-Training & 30 & $36.5 \%$ & 45 & $54.8 \%$ & 7 & $8.7 \%$ & 0 & 0 \\
Time Arrangement & 36 & $43.9 \%$ & 38 & $46.3 \%$ & 6 & $7.3 \%$ & 2 & $2.5 \%$ \\
Special Lecture & 17 & $20.7 \%$ & 28 & $34.2 \%$ & 36 & $43.9 \%$ & 1 & $1.2 \%$ \\
Course Content & 40 & $48.7 \%$ & 29 & $35.3 \%$ & 13 & $16 \%$ & 0 & 0 \\
Appearances & 62 & $75.6 \%$ & 20 & $24.4 \%$ & 0 & 0 & 0 & 0 \\
Visit Activities & 65 & $79.2 \%$ & 14 & $17 \%$ & 3 & $3.8 \%$ & 0 & 0 \\
\hline
\end{tabular}

3) “Pre-job training activities have what it takes to increase?” For the majority of respondents believe that this problem can increase the interaction of cultural and sports activities, practical activities, a variety of activities to enhance teamwork, and build a new information exchange and other groups of workers.

\section{Discussion}

\subsection{The Overall Arrangement of Pre-Service Training}

The pre-job training for new employees is focusing on teaching, mainly in the form of seminars [8] [9]. Thematic content is divided into 11: the historical and cultural themes hospitals, medical safety and quality topics, topics Medical Service, Personnel topics, quality and safety supervise topics, nursing topics, Dean Topics, research at topics, medical technology topics, network and healthcare topics, safety education topics. Trainers are held by the House leadership and the various departments responsible person. Training schedule is in late July, the second day, the lead organization carried out by the Human Resources Department after reports on new hires. The first week is focused on pre-service training, every Thursday night before to arrange a training seminar, the strict attendance and attendance system as a payroll basis.

\subsection{Employees Have Less Role Perception}

Role of cognitive character creation is the first stage, the new workers into the role as the actor must be based on their own perception of the role played by the premise, in order to better stage roles converted into the behavior. Each individual has a role to cognitive adaptation period, if longer adaptation period will be a variety of serious problems [10] [11]. The aim of our pre-service training for new employees is to try to shorten the adaptation period for the new role. The main reason for the lack of staff role perception there are two, one is the pre-service training of the employer is not detailed enough, the lack of specific guidance staff. Another is the role of cognition there are individual differences, each employee understanding and awareness of the role are different. For faster shorten new staff on the role of adaptation period, pre-job training should be more detailed work. Before statistics can be seen in different departments categories of employees with different educational topics favorite courses are not the same, indicating that demand for new workers in the pre-job training is not the same. Before the training of new employees basic conditions for serious research and analysis is to carry out taxonomic training for different jobs, education. Let the work of a different nature, different levels of learning ability of new employees can be different according to their specific circumstances and professional characteristics of the work there is a clearer role to play in the future, an accurate perception.

\subsection{Enhance Staff Self-Awareness}

Everyone should re-examine in entering a new environment when their own, recognize themselves in the new environment of another, in this period will inevitably bring a character to the next of them [12]. For example, before most of the new plays the role of student workers, after the sudden will to work on the job performance of the students still like. For their own lack of knowledge and positioning is not accurate, leading employees can not face their own advantages and disadvantages, blindly think they mastered the theoretical knowledge can be a good job, the lack of practical experience and hard-working spirit, into self-awareness Mistakes. Employee selfawareness is not sufficient is not conducive to future performance at work among. To address this issue in the 
pre-service training courses should add more interactive content, a single theory too boring lecture form, cannot effectively help employees complete the transition from student to the community on self-awareness. Curriculum design and training form appropriate diversification, the use of multimedia tools to try some new teaching methods, such as for employees to participate in the role-playing simulation scenarios, case studies, field visits and exercises. Topics such as safety education courses in fire safety education, not just merely arranged for staff in the classroom watching the video, can arrange a field of fire scenario exercises. More staff to the field to visit various departments, simulated work scenarios, new employees can not only enhance the enthusiasm of the enthusiasm and let them know more about the hospital from the senses, the ability to increase teamwork and understanding. So that the new workers from a single theory of knowledge and learning, hands-on activities in their ability to have a clearer understanding examine their shortcomings and make improvements, and accumulated practical experience better into future work.

\subsection{The Conversion of Staff Exchanges and Expands Information Feedback}

In order to continuously improve the quality of pre-service training to help new employees faster and better fulfill their role of conversion, it is also important to collect the information and views on the experience of staff training. After completing pre-service training, we then carry out follow-up counseling work, such as long-term continuous staff training to hold a new experience exchange meeting on a regular basis, the use of a variety of today's popular chat software (such as QQ or micro-letters, etc.) to establish after-school exchange and communication of the staff and help them solve their problems that are encountered in role reversal. Jointly with other agencies or organizations, the development training will deepen the effect of the training. The multi-organization spare time sports activities enrich their collective life, promote communication between employees, strengthen the emotional between employees, enhance interpersonal communication skills so that new staff have better understanding of the hospital's culture, more quickly adapt into the hospital's working environment and their new roles and the conversion of the roles is completed [13].

\section{Conclusion}

Through this survey of pre-job training, to understand the different positions, different academic staff for training satisfaction, as well as the problems and shortcomings, we learned their different needs and combined with social role theory direction for future training proposals. For the different professional characteristics, the nature of work and the qualifications of the staff are more refined and targeted to the preparation of training courses, formal training improves inter-activity, increases opportunities for employees in the field and hands-on involvement. After-school staff communication platform is established to expand the training to deepen and strengthen the emotional communication between employees, to promote the hospital culture and to ensure the quality of training. The establishment of the platform also lays a solid foundation for faster and better help of new staff to complete the role reversal, the hospital personnel training, cultural construction and sustainable development escort.

\section{Acknowledgements}

The authors thank Chongqing Medical University for excellent assistance. The study was supported by Humanity and Social Science of Chongqing Education Committee (15SKG025), Chongqing Academy of Social Science, and Chongqing Science \& Technology Commission (2013YBGL138, 20130115, 2012-2-017). The authors also thank National Institute of Health (NIH) [(R01) RFA-WT-003].

\section{References}

[1] Wang, J. (2012) Effectiveness of Human Resources Training for SMEs. Human Resources Management, No. 6, 39-40.

[2] Dong, M.H., Niu, X. and Li, S.L. (2012) On the Perspective of the New Employees How to Improve the Effect of PreService Training. Modern Enterprise Education, No. 2, 34-36.

[3] Bao, W. (2011) Reflections on Pre-Service Training of Hospital. China Practical Medicine, 6, 274-275.

[4] Wang, Q., Ju, H.Y., Miao, M., et al. (2010) New staff Pre-Job Training in the Form of Survey Results. Modern Hospital, 10, 124-126. 
[5] Sun, J.L. (2010) Talking about the Significance of Pre-Service Training of Medical Graduates. Medicine Information, 23, 604-605.

[6] Ji, X.Q. and Gui, T.F. (2009) Practical Experience of Pre-Job Training of New Employees. Modern Hospital, 9, 112113.

[7] Yang, S.H. (2009) How Do the New Hospital into Pre-Service Training of Medical Staff. Modern Economics, 8, 84-85.

[8] Wang, X. (2007) The Key Step in the Training of New Employees: Employee Orientation. China Human Resources Development, No. 97, 43-46.

[9] Liu, L. and Jing, Z.Y. (2007) Thoughts on College Students Hospital Training before the New Assignment. Chinese Health Service Management, 23, 303-304.

[10] Gao, G.J. (2007) Analysis and Reflections on Students' Career Planning. Chinese High Education, No. 7, 47-48.

[11] Xi, C.Q. (2010) Role Theory. Zhejiang University Press, Hang Zhou.

[12] Wang, R.H. (2007) Human Behavior and Social Environment. 2nd Editorial, East China University of Technology Press, Shanghai.

[13] Li, Y. (2011) Introduction to Sociology. Jinan University Press, Guangzhou. 